\title{
ISCT survey on hospital practices to support externally manufactured investigational cell-gene therapy products
}

\author{
Alexey Bersenev ${ }^{1,2, *}$, Michael P. Gustafson ${ }^{1,3}$, Patrick J. Hanley ${ }^{1,4}$ \\ ${ }^{1}$ Immuno-Gene Therapy Committee, International Society for Cell and Gene Therapy, Vancouver, BC, Canada \\ ${ }^{2}$ Department of Laboratory Medicine, Yale School of Medicine, Yale University, New Haven, Connecticut, USA \\ ${ }^{3}$ Department of Laboratory Medicine and Pathology, Mayo Clinic in Arizona, Phoenix, Arizona, USA \\ ${ }^{4}$ Center for Cancer and Immunology Research, Children's National Hospital, and the George Washington University, Washington, DC, USA
}

\section{A R T I C L E I N F O}

\section{Article History:}

Received 27 July 2021

Accepted 13 September 2021

\section{Keywords:}

CAR T

cellular therapy

gene therapy

immunotherapy

\begin{abstract}
A B S T R A C T
There is considerable interest in the next generation of personalized medicine, especially cell and gene therapy products such as chimeric antigen receptor T cells (CAR-Ts). Unlike other small molecules or pharmacologic drugs, most existing cell or cell-based gene therapy products (CGTs) require apheresis collection of the patient or donor, subsequent manufacture of the product, and final shipment of the product to the clinical site for infusion. Whereas traditional pharmaceutical drugs have involved the drug sponsor and the clinical site and clinical pharmacy, this new manufacturing paradigm has evolved, in many cases, to include an apheresis center, a cell processing lab, the sponsor's manufacturing facility, and a clinical site with or without a pharmacy. Here we report the results of a survey of current practices handling investigational CGTs conducted by the Immuno-Gene Therapy committee of the International Society of Cell and Gene Therapy.
\end{abstract}

(C) 2021 International Society for Cell \& Gene Therapy. Published by Elsevier Inc. All rights reserved.

\section{Introduction}

Since the first approval of a cell-based gene therapy product for cancer in 2017 (Kymriah: anti-CD19 chimeric antigen receptor T cells), four additional products have been approved for cancer. All of these products have the same manufacturing paradigm: an apheresis starting material is collected (typically at the hospital's apheresis collection site), and the material is shipped fresh or frozen to the manufacturing site, where it is manufactured, tested and cryopreserved. The final drug product is then shipped back to the hospital, typically to the cell processing lab (CPL) or in some cases the hospital's cell pharmacy or clinical program. This is in stark contrast to traditional pharmacotherapies, which are prescribed by a physician and distributed by the pharmacy to clinicians. Hospital-based CPLs have been manufacturing hematopoietic progenitor cell (HPC) products for decades with varying complexity, but these minimally manipulated products (often called "361 products" after section 361 of the Public Health Service Act) typically have been manufactured completely in-house and then distributed to the clinical team for infusion. Although there are only six of these approved drugs at the moment, hundreds of industry-sponsored studies are in clinical trials that fit this paradigm. The advent of more-than-minimally manipulated products, which are regulated under section 351 of the Public

\footnotetext{
* Corresponding Author: Department of Laboratory Medicine, Yale School of Medicine, Yale University, 20 York St, PS 329E, New Haven, CT 06510.

E-mail address: alexey.bersenev@yale.edu (A. Bersenev).
}

Health Service Act, has created new challenges related to handling of cell or cell-based gene therapy products (CGTs) that are manufactured by third-party organizations (industry or academic). To address how the cell and gene therapy community is working to meet the new demands of industry-sponsored investigational products, the Immuno-Gene Therapy Committee of the International Society for Gene and Cell Therapy (ISCT) conducted a survey on current practices for handling investigational CGT products at the point of patient care. The objective of this survey was to gather information and to inform the professional cell therapy community about different practices across multiple laboratories/institutions.

\section{Methods}

A freely available web-based platform (Google Forms) was used to conduct the survey. The survey was distributed among the professional community via (i) Small Cell Therapy Lab (Google Group), (ii) laboratory members of ISCT and (iii) personal emails. The survey included 11 questions and was open online for 2 months.

The scope of the survey included the following:

a. Investigational products (under the Food and Drug Administration's [FDA's] Investigational New Drug [IND] status). Information related to handling of commercial CGTs (such as CAR-T cell therapy) was outside of the scope of this survey.

b. The survey covered CGTs regulated as human cells, tissues, and cellular and tissue-based products (HCT/Ps) according to section 
351 in the US and advanced therapy medicinal products (ATMPs) in Europe, as well as analogous products, developing in other jurisdictions, that required clinical trials.

c. Externally manufactured CGTs (by third-party organizations). Institute/hospital CGTs (developed in-house) were outside of the scope of this survey.

\section{Data collection and analysis}

Data were collected via Google Forms and exported to Microsoft Office Excel. The survey was not anonymous. The name of the participating institution and email of responder were included in the survey for better analysis and validity. Data analysis was performed in Excel by one assessor (A. Bersenev) and validated by two other ISCT committee members (P.J. Hanley and M.P. Gustafson).

\section{Results}

We received 42 responses (36 unique and 6 duplicate) from February 4 to April 5, 2021. All 36 unique hospital laboratories/institutions were included in further analysis. In terms of geographical distribution of responding laboratories, only four countries were represented, with the vast majority of responses (83\%) from the United States (Figure 1).

To determine how CPLs were organized to handle externally manufactured CGTs we asked each site to choose the structure that best fits their organization. Six different scenarios were designed to handle externally manufactured CGTs. The majority of respondents (78\%) were organized as a stem cell processing laboratory, serving both externally manufactured products and the hematopoietic stem cell transplant program (Table 1). To estimate the volume of externally manufactured CGTs, we next asked how many accruing clinical trials each institution is currently handling and how many additional clinical trials were planned in the next 6-12 months. There was a wide range in responses, but the majority of institutions (67\%) currently support one to 10 clinical trials, with only one institution supporting $>35$ (Figure 2). Given the rapid growth in the field of CGT, we next asked survey participants to indicate the number of trials that involved externally manufactured CGTs that they expect to support in the next 6-12 months. About half of all responding facilities planned to add one to five additional trials in the next 6-12 months (Figure 3).

Processing externally manufactured CGTs takes a considerable amount of time by various staff in the facility; however, this time is relatively easy to account for. One underappreciated aspect of these trials that is more difficult to quantify is the time and effort spent on agreements between the sponsor and the institution. Whether the agreement is the primary responsibility of the clinical team, the CPL,

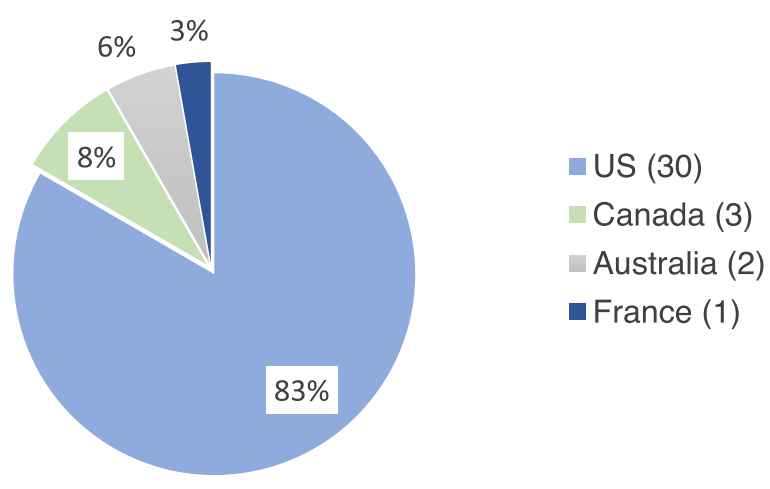

Figure 1. Geographic distribution of responding laboratories. Survey participants were asked to indicate the location of the CPL. (Color version of figure is available online).
Table 1

Structure of CPLs supporting externally manufactured CGTs

\begin{tabular}{ll}
\hline Element & $\mathrm{n}(\%)$ \\
\hline Stem Cell Processing Laboratory (also serves HPC Transplant Program) & $28(78)$ \\
Advanced Cell Therapy Laboratory (GMP Facility) & $2(5)$ \\
Two to three units combined under one leadership and quality system & $2(5)$ \\
Separate unit with dedicated personnel & $1(3)$ \\
Other: both Stem Cell Processing and Advanced Cell Therapy & $2(5)$ \\
$\quad$ Laboratories & $1(3)$ \\
Other: Clinical Islet Laboratory & 0 \\
Hospital Pharmacy & \\
\hline
\end{tabular}

or another unit of the hospital also varies between institutions. Therefore, we next surveyed whether hospital facilities have agreements that describe services they provide to handle externally manufactured CGTs, and with whom these agreements reside. Thirty-five respondents indicated that there are a variety of ways to cover hospital facility services, including the use of agreements and/or memoranda of understanding (MOUs) (Figure 4). Interestingly, 37\% of respondents indicated that the $\mathrm{CPL}$ is not the primary unit responsible for the agreements, whereas 37\% indicated that the CPL was the primary unit responsible for the agreement with either the sponsor, clinical trial unit, or study principal investigator (PI).

In addition to agreements, a number of other responsibilities are difficult to quantify and capture for revenue purposes. Examples of these include time spent onboarding a clinical trial (reviewing standard operating procedures [SOPs], the protocol, emailing the sponsor, meeting with the sponsor, creating new forms or SOPs), audits, product handling (receipt, storage, etc.), and product infusions. Institutions/CPLs have a number of mechanisms to charge (or not charge) for these services. To capture this fee structure, survey respondents were asked to indicate how fees were charged. Most respondents indicated that all these items were included in their fee structure (23 of 31 respondents, $74 \%$ ), whereas $16 \%$ ( 5 of 31 responders) indicated they charged only for product handling. Given the report from Digiusto et al. [1] describing various business models for CPLs, it is enlightening to observe that many fees are not being appropriately captured.

As mentioned earlier, one of the challenges with the rapidly growing CPLs is supporting the increasing numbers of clinical trials and the non-manufacturing time that it takes to onboard these studies. In the survey, we asked participants to indicate whether they negotiated the service fee (where "fee" was not specifically defined) and the frequency with which this occurs. About half of all responders indicated that they were able to successfully negotiate their fees; the other half indicated that they negotiate rarely, with no changes, or they never were involved in negotiations (Figure 5).

At the Academic GMP [good manufacturing practices] Symposium held in 2018, a similar survey asked participants how the CPLs were funded [1]. To this end, we were interested in ascertaining whether facilities were receiving financial support from their institution/hospital as a response to an increase in industry-sponsored cell or cellbased gene therapy clinical trials. Most responders (23 of 35) indicated that their financial support is not bad but could be improved, whereas $17 \%$ of facilities (6 of 35) indicated that they are getting poor financial support (Figure 6).

Beyond institutional financial support, we also wanted to learn more about how well institutions supported CPLs when it came to hiring new personnel, and how many staff the CPLs have added to accommodate the boom in cell or cell-based gene therapy clinical trials. Most respondents (57\%) indicated that although staffing support for new hires is reasonable, it is slow and needs improvement; $31 \%$ of respondents indicated that they do not receive enough institutional support for hiring new personnel (Figure 7). When we surveyed how many staff were added to the program to accommodate new clinical 


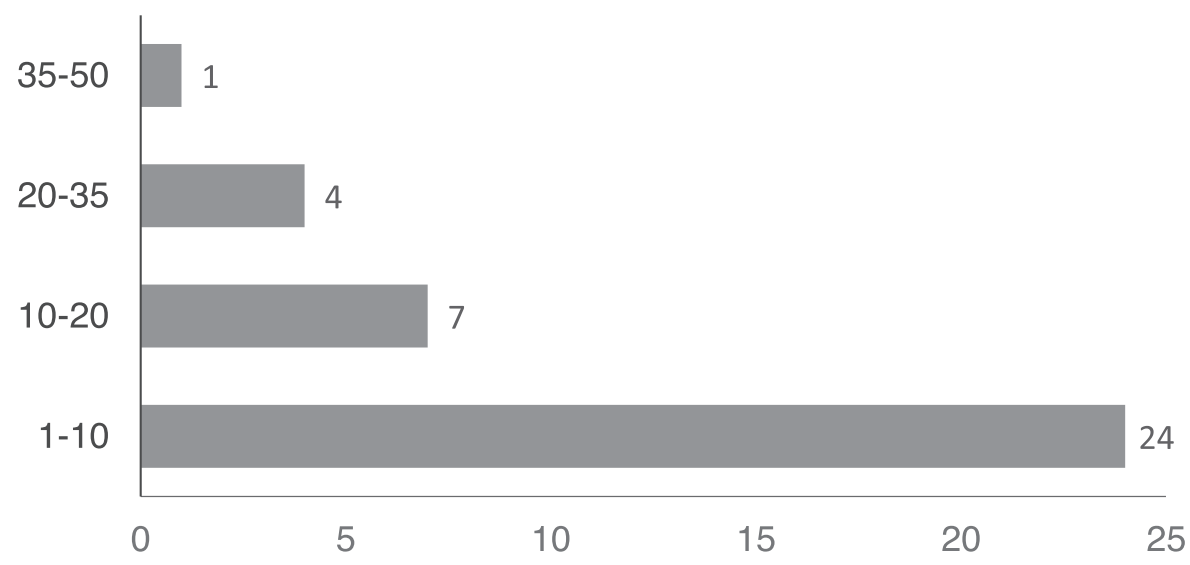

Figure 2. Number of CGT clinical trials supported by CPLs.

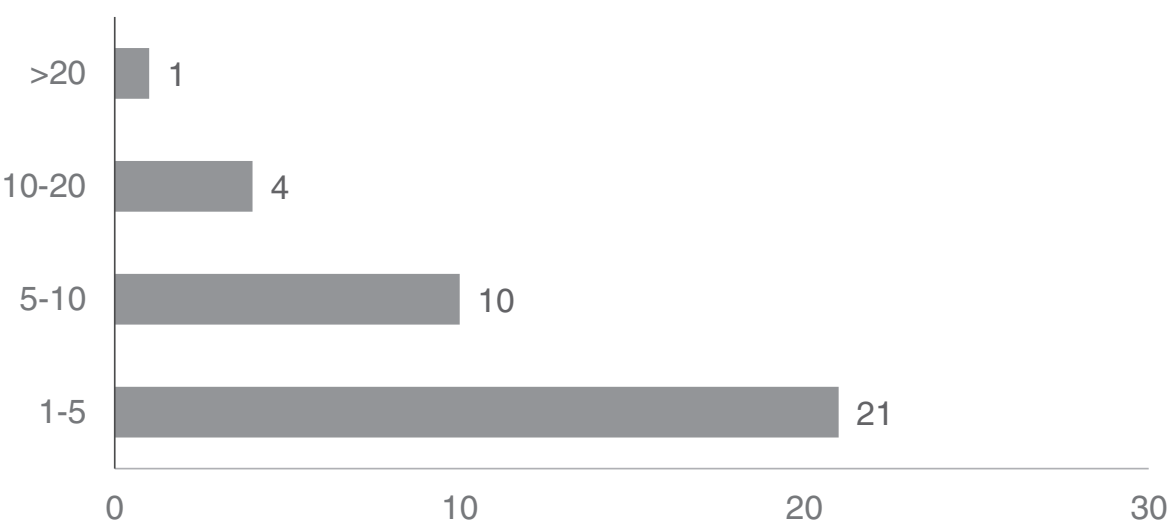

Figure 3. Number of additional trials projected in the next 6-12 months.

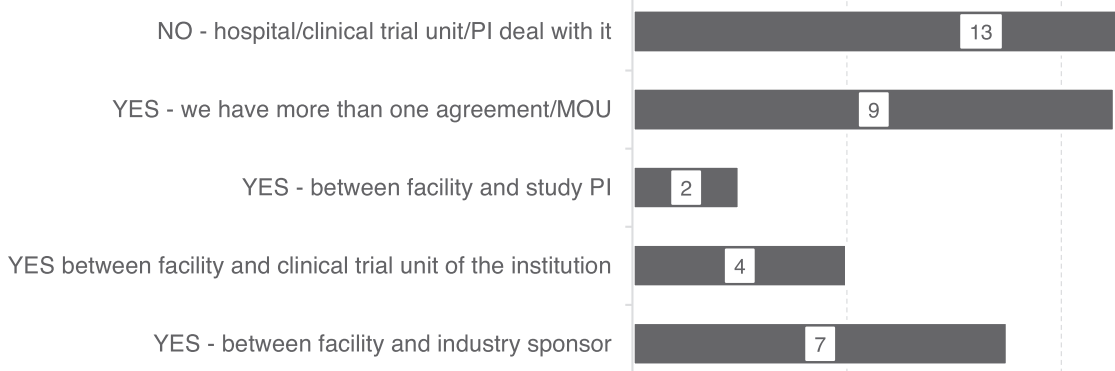

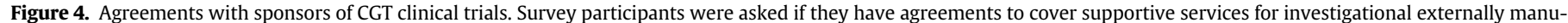
factured CGTs.

NO - sometimes explain charges, no fees change

NO - never had such experience

YES - rarely, fees change

YES - frequently, fees change

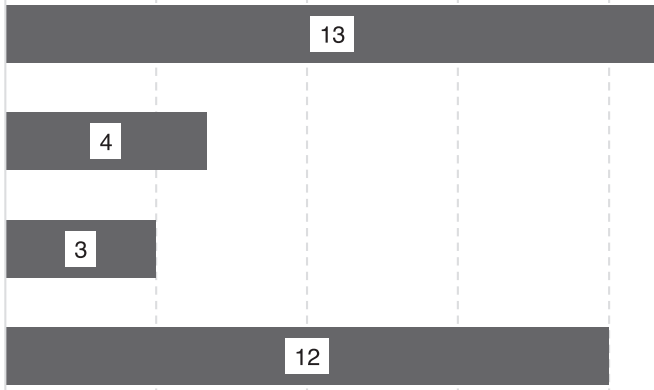

Figure 5. Negotiation of service fee. 


\section{$\because$ YES $\quad$ NO $\quad$ OK, but could be imporved}

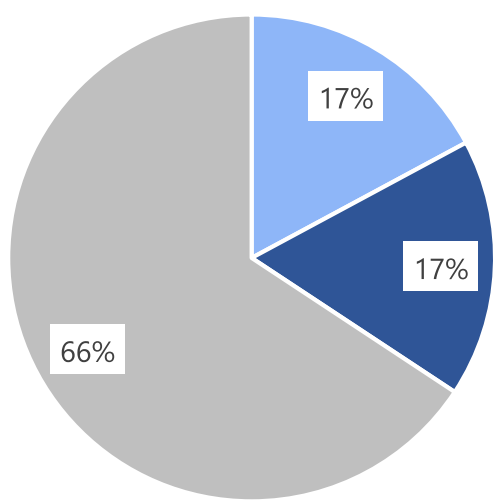

Figure 6. Institutional support of CPLs. Survey participants were asked if they have enough financial support. (Color version of figure is available online).

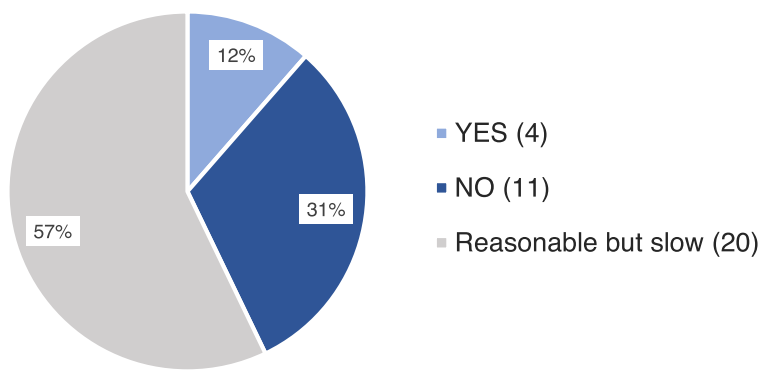

Figure 7. Institutional support for hiring new staff. Survey participants were asked if they have enough institutional support for hiring of new personnel. (Color version of figure is available online).

trials, $46 \%$ said that they did not add any new staff, and $41 \%$ said they added one to three new staff members to accommodate the new trials. For more information about staffing, we asked how many new staff members were hired to support activities related to handling investigational externally manufactured CGTs (Figure 8).

We realized that an influx of trials involving novel, externally manufactured CGTs may be associated with creating new or changing existing laboratory policies and procedures. Thus we asked how facilities address this new challenge (see Table 2). The vast majority of facilities incorporated new policies and procedures and are continuing to do so. The next question was to clarify how facilities incorporating new policies and processes (see Table 3). Multiple approaches were mentioned by respondents, but most involved creating new or changing existing SOPs and referencing sponsor-provided manuals.

\section{Discussion}

This was the first attempt by a professional society to learn how hospital-based CPLs are accommodating a rapidly growing number of

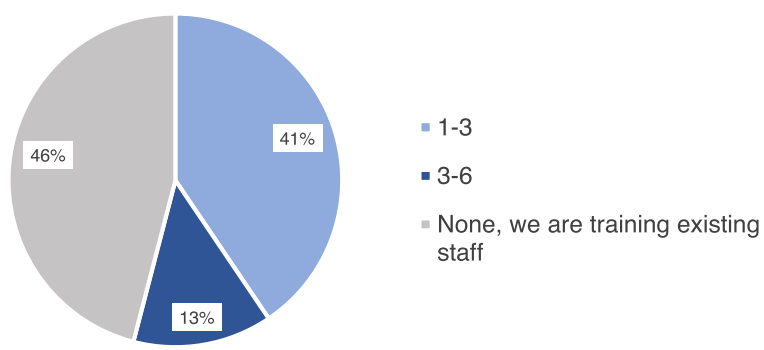

Figure 8. Number of staff added to accommodate new CGT trials. (Color version of figure is available online).
Table 2

Is your facility incorporating new policies/procedures related to investigational externally manufactured CGT products?

\begin{tabular}{ll}
\hline Response & $\mathrm{n}(\%)$ \\
\hline $\begin{array}{l}\text { No, our internal policies/procedures are sufficient } \\
\text { Yes, we are constantly incorporating new procedures/processes in our } \\
\text { practice }\end{array}$ & $5(14)$ \\
$\begin{array}{l}\text { Yes, we incorporated some new procedures at the beginning when } \\
\text { unit was established }\end{array}$ & $10(28)$
\end{tabular}

clinical trials in which investigational CGT products are manufactured externally. In this survey, the majority of respondents were from North America and currently supported one to 10 CGT clinical trials. Respondents also indicated that most agreements are dealt with by the facility itself rather than other units of the hospital, and most did not negotiate for service fees. Sixty-eight percent of CPLs said that institutional support was okay but could be improved; $46 \%$ stated that they did not plan to hire additional staff, whereas $41 \%$ said they hired one to three staff members to accommodate the increase in externally manufactured CGT clinical trials. Overall, these responses demonstrated similar trends within CPLs in how they sought to address the increased volume of clinical trials.

This particular question-how CPLs accommodate the increased volume-captured our attention specifically because this is a very new area of medical research that requires the support of highly specialized hospital/academic-based facilities and has required the creation of infrastructure that did not previously exist. Although regulations and standards exist for these types of products [2], they are still relatively new, they are not widespread, and even the biggest, most advanced centers may treat only a thousand patients a year with cellular therapy products (and the majority treat far fewer).

When deciding on how to focus this survey, we deliberately chose to exclude commercially available products and internally manufactured products. We did this because commercially available products are more standardized and have well-defined regulatory requirements (for example, the Risk Evaluation and Mitigation Strategy [REMS] programs that are currently required by the FDA for commercial CAR-T products). Because they are licensed drugs, the costs associated with them are also straightforward. Investigational CGTs, on the other hand, require a number of regulatory approvals such those required by the FDA (IND application), investigational review board and institutional biosafety committee.

Assessing the costs associated with supportive services for these products is also less straightforward, as they can involve start-up fees, new protocols, new validations, new training and costs for audits and administrative time; these charges are more challenging to account for and, as demonstrated in the survey, are not always included in the final invoice. We did not ask whether CPL charges

Table 3

How are you incorporating new requirements and differences between multiple externally manufactured CGT products?

\begin{tabular}{|c|c|}
\hline Response & $\mathrm{n}$ \\
\hline We are only using manuals provided by sponsor/manufacturer & 4 \\
\hline $\begin{array}{l}\text { We are writing our own new SOPs, based on manuals provided by } \\
\text { sponsor and training }\end{array}$ & 17 \\
\hline $\begin{array}{l}\text { We are revising our existent SOPs to accommodate all differences } \\
\text { between products }\end{array}$ & 5 \\
\hline We are performing validations to incorporate novel procedures & 0 \\
\hline $\begin{array}{l}\text { We are referencing sponsor's manuals in our SOPs/document control } \\
\text { sponsor's manuals }\end{array}$ & 2 \\
\hline $\begin{array}{l}\text { We combine study manuals with our batch record, adjusted to specific } \\
\text { study from the basic template }\end{array}$ & 1 \\
\hline $\begin{array}{l}\text { We created an overall clinical trial SOP to cover special needs outside } \\
\text { of routine; we created reference Job Aids for every clinical trial } \\
\text { which reference study SOPs }\end{array}$ & 1 \\
\hline
\end{tabular}


were a part of the overall institutional/hospital budget to support the whole clinical trial, and therefore, it is unclear whether these charges had any financial impact on the facility. In a 2018 survey of academic GMP facilities [1], DiGiusto found that an average of $\sim 22 \%$ of facilities received institutional funding, with another 20\% receiving philanthropic funds. Unlike academic GMP facilities, which are mostly dedicated to internally manufactured CGT products, hospital CPLs may have a different funding structure. We think that the service fee for support of externally manufactured investigational products by CPLs may create a new and unique revenue flow and, if directed properly by the hospital, could support CPL operations and improvement.

Lastly, the sheer number of investigational CGTs means that there is inherent variability and uncertainty that are difficult to control for each of the CPLs. Internally manufactured products were excluded because they are typically investigator-initiated products that are home grown and hence have less variability and require less training, and CPLs have more control over the process.

The survey has provided visibility into how CPLs have evolved to accommodate novel CGT clinical trials, but it has a number of caveats. First, it is relatively small (36 responding facilities) and skewed toward North America (92\% of respondents located in North America). Investigational CGTs are not limited to North America and are currently being tested on almost every continent. The diverse health care systems across the world may significantly affect how other regions evolve to accommodate these products. Nevertheless, taking into consideration a larger involvement of hospital pharmacists in handling of commercial CAR-T cell products in Europe [3] we would be interested in learning more about European practices in handling externally manufactured CGTs by the hospitals. Second, although we felt it was necessary to focus on investigational CGTs, it was difficult to set a clear definition of investigational CGTs that are externally manufactured and also capture all differences with commercial and in-house-developed CGTs. So, while many CPLs may have made changes to their program to accommodate new CGTs, it is difficult to uncouple whether those CGTs are investigational or commercial-or in some cases even internally manufactured. Third, the survey did not capture all aspects and variabilities related to practices of handling externally manufactured investigational CGTs, including the size of the laboratory (which would largely skew how they are organized to handle these products and the number of trials or staff they have), how the laboratory is organized (and the position of the respondent in the lab), and the experience of the laboratory.

Finally, the survey itself had some shortcomings, namely that the categories may have caused some confusion ( $1-10$ versus $10-20$, for example), and the duration of some of the questions was not indicated or not clear, which could have unintentionally skewed the results. For instance, in Figure 3, it was indicated that in 6-12 months, the majority of CPLs planned to add one to five clinical trials. If that timeline were extended to 2-3 years, the number of planned trials may be significantly higher.

In spite of these caveats, this survey, which to our knowledge is the first to be reported, is a useful resource for programs building CPLs and looking to accommodate the rapid surge in investigational CGTs. Based on the results, there are areas of growth such as agreements and budgets that have limited consensus. With time, we expect that these will become more standardized, ultimately providing as much revenue and support as possible to provide justification for the increase in staff needed to support the increased volume of patients and clinical trials.

\section{Conflict of Interest}

P.J. Hanley is a co-founder and on the board of directors of Mana Therapeutics and serves as an advisor to Cellevolve and Maxcyte. A. Bersenev is a co-founder of Cell Trials Data and co-founder and Managing Director of CellBioEngines.

\section{Acknowledgments}

The authors thank the Immuno-Gene Therapy Committee of the International Society of Cell and Gene Therapy for their contributions and review of this manuscript.

\section{Supplementary materials}

Supplementary material associated with this article can be found in the online version at doi:10.1016/j.jcyt.2021.09.009.

\section{References}

[1] Digiusto DL et al. Proceedings of the first academic symposium on developing qualifying and operating a cell and gene therapy manufacturing facility. Cytotherapy 2018;20:1486-94.

[2] Standards for Immune Effector Cells: Foundation for the Accreditation of Cellular Therapy; 2020 Available from: http://www.factwebsite.org/iecstandards/

[3] Marzal-Alfaro María Belen, et al. Chimeric Antigen Receptor T Cell Therapy Management and Safety: A Practical Tool From a Multidisciplinary Team Perspective. Front Oncol 2021;11:636068. 